\title{
New Stability Conditions for Nonlinear Systems Described by Multiple Model Approach
}

\author{
Ameur Sassi, Afef Abdelkrim \\ Laboratoire de recherche en automatique (LA.R.A), National School of Engineers of Tunis (ENIT), Tunisia
}

\begin{tabular}{l}
\hline Article Info \\
\hline Article history: \\
Received Aug 17, 2015 \\
Revised Oct 19, 2015 \\
Accepted Nov 1, 2015 \\
\hline
\end{tabular}

\section{Keyword:}

Electrical circuit

Linear matrix inequalities

Multiple model

Nonlinear system

Quadratic Lyapunov function

\begin{abstract}
This paper studies the global asymptotic stability and the tracking control problem of an uncertain non stationary continuous system described by the multiple model approach. It is based on the construction of a basis of models containing four extreme models and possibility of addition of an average model. Once the basis of models is generated, an operation of fusion of these different models is made to the level of the elementary control law and the partial output using the geometric method. New sufficient conditions for the stability are derived via Lyapunov technique. The matrices of feedback gains and tracking gains are determined while solving systems of LMI constraints (Linear Matrix Inequalities). The case of an unstable continuous nonlinear model of electrical circuit operating in pseudo-periodic system is considered to illustrate the proposed approach.
\end{abstract}

Copyright (C) 2016 Institute of Advanced Engineering and Science. All rights reserved.

\section{Corresponding Author:}

Ameur Sassi,

Laboratoire de Recherche en Automatique (LA.R.A),

Ecole Nationale d’Ingénieurs de Tunis, BP 37, 1002 Tunis Belvédère, Tunisia

Tel : +216 71874 700, Fax: +216 7182729

Email: Ameur.Sassi@enit.rnu.tn

\section{INTRODUCTION}

The multiple model approach is proving very interesting whenever we are confronted with complex systems and/or nonlinear. It is to represent the system studied by a family of simpler and easier to manipulate mathematical models [1], [2], [3]. This approach has been recently developed in several science and engineering domains, with typical applications in the electrical and mechanical engineering areas, with application to modelling, control and/or fault detection. It was introduced as an efficient and powerful method to cope with modelling and control difficulties when complex non linear and/or uncertain processes are concerned. The multiple model approach assumes that it is possible to replace a unique non linear representation by a combination of simpler models thus building a so-called model-base. Usually, each model of this base describes the considered process at a specific operating point [4]. The interaction between the different models of the base through normalized activation functions allows the modelling of the global nonlinear and complex system. The stability of these models is, most of the time, studied using the quadratic Lyapunov approach [5], [6], [7], [8], [9], [10]. The obtained conditions are given in terms of Linear Matrix Inequalities (LMI) and can be efficiently solved by convex programming techniques [11], [12], [13], [14].

The stability conditions based on the use of the quadratic Lyapunov function are conservative as a single common symmetric positive definite matrix verifying all Lyapunov inequalities is required [13]. It is also rejected by certain systems such as the saturated systems, the piecewise linear systems, etc. Some works show the contribution of the polyquadratic and the piecewise quadratic Lyapunov functions, [15], [16], [17].

The study proposed in this paper focuses on a class of uncertain systems and complex continuing bounded parameters [18], [19]. The global model can be obtained either by using the switching operation or fusion. In this study, we are interested in the fusion using the geometric method [19]. 
This method is seen at both the elementary controls at the level of partial outputs and driven by validity indices.

Our new stability conditions are proposed in the case of multiple model stabilizing state feedback control with reference, permits to locally find a symmetric positive definite matrix to each model of the basis. At the entrance to each base model in closed loop control is applied overall inferred weights of elementary linear feedback control with reference state and to converge the global output of the controlled system to a desired trajectory. Matrices gains by state feedback and tracking are determined by solving systems stability conditions given in terms of LMI [20], [21], [22], [23].

This paper is organized as follows. In Section 2, we introduce briefly some basic notions of multiple model appraoch and the theoretical tool used in this work, namely the algebraic method. In section 3, the strategies of multiple model control based on geometric method is proposed. Stability conditions using new LMI approach based on state feedback with reference are provided in section 4 . In the section 5 an example of an unstable second order continuous model of electrical circuit operating in pseudo-periodic system is is studied to illustrate the efficiency of this apprach. Conclusion is drawn in section 6 .

\section{Notations}

- $\quad$ The symbol $(*)$ denotes the transpose elements in the symmetric positions, for example,

$$
X+(*)<0 \Leftrightarrow X+X^{T}<0 \text { and }\left(\begin{array}{cc}
A & B \\
(*) & C
\end{array}\right)<0 \Leftrightarrow\left(\begin{array}{cc}
A & B \\
B^{T} & C
\end{array}\right)<0
$$

- $\quad$ LMI: Linear Matrix Inequalities

\section{PROBLEM STATEMENT}

The evolution of a continuous non-stationary uncertain parameters and bounded complex system is described by the following differential equation:

$$
a_{0}(.) y(t)+a_{1}(.) y^{(1)}(t)+\ldots+a_{n-1}(.) y^{(n-1)}(t)+y^{(n)}(t)=u(t)
$$

with the symbol (.) represents the set of variables, uncertainties, noise or disturbances acting on the coefficients of this system as, for: $i=0,1, \cdots, n-1, \underline{a}_{i} \leq a_{i}(.) \leq \bar{a}_{i}$ with $\underline{a}_{i}=\min _{i}\left(a_{i}().\right)$ and $\bar{a}_{i}=\max _{i}\left(a_{i}().\right)$. Model (1) may be given in the following controllability companion form [15]:

$$
\left\{\begin{array}{l}
\dot{x}(t)=A(\cdot) x(t)+B u(t) \\
y(t)=C x(t)
\end{array}\right.
$$

where $A, B$ and $C$ are respectively the state matrices, control and output with:

$$
A(\cdot)=\left(\begin{array}{ccccc}
0 & 1 & 0 & \ldots & 0 \\
\vdots & 0 & 1 & & \vdots \\
& \vdots & 0 & \ddots & \\
& & \vdots & \ddots & \\
0 & 0 & 0 & & 0 \\
-a_{0}(.) & -a_{1}(.) & -a_{2}(.) & \ldots & -a_{n-1}(.)
\end{array}\right), B=\left(\begin{array}{lllll}
0 & \ldots & 0 & 1
\end{array}\right)^{T} \text { and } C=\left(\begin{array}{llll}
1 & 0 & \ldots & 0
\end{array}\right)
$$

The state matrices characterizing the four models $M_{i}$ of the base are given by $A_{1}\left(\underline{a}_{0}, \underline{a}_{1}, \bar{a}_{2}, \bar{a}_{3}, \ldots\right), A_{2}\left(\underline{a}_{0}, \bar{a}_{1}, \bar{a}_{2}, \underline{a}_{3}, \ldots\right), A_{3}\left(\bar{a}_{0}, \bar{a}_{1}, \underline{a}_{2}, \underline{a}_{3}, \ldots\right)$ and $A_{4}\left(\bar{a}_{0}, \underline{a}_{1}, \underline{a}_{2}, \bar{a}_{3}, \ldots\right)$.

In addition to these models, it is worth adding the average model as a fifth in the database, it somehow represents the barycentre of the extreme models and parameters are defined by the arithmetic mean of the four parameters extreme models with $a_{5 i}=\frac{\underline{a}_{i}+\bar{a}_{i}}{2}$ for $i=0,1, \cdots, n-1$. 
A comprehensive description of the studied system can be defined from an interpolation model $M_{i}$ based on the following state representation [18]:

$$
\left\{\begin{array}{l}
\dot{x}(t)=\sum_{i=1}^{r} \lambda_{i}(t)\left(A_{i} x(t)+B_{i} u(t)\right) \\
y(t)=\sum_{i=1}^{r} \lambda_{i}(t) C_{i} x(t)
\end{array}\right.
$$

where $A_{i}, B_{i}$ and $C_{i}$ are respectively the state matrices, control and output of each of the $M_{i}$ basic model.

In this paper, the main objective is to converge the output $y(t)$ of the global system (3) to a desired trajectory $y_{d}(t)$, i.e., $y(t)-y_{d}(t) \rightarrow 0$ as $t \rightarrow+\infty$ with:

$$
y_{d}(t)=\sum_{i=1}^{r} \lambda_{i}(t) C_{i} x_{d}(t)
$$

$x_{d}(t)$ is the desired state vector.

\section{MULTIPLE MODEL CONTROL STRATEGY USING GEOMETRIC APPROACH}

The geometric method is used to calculate the distance $d_{i}(t)$ between the partial outputs $y_{i}(t)$ and the desired trajectory $y_{d}(t)$, such as [10],[11]:

$$
d_{i}(t)=\left|y_{d}(t)-y_{i}(t)\right|
$$

The normalized distance $n_{i}$ is given by:

$$
n_{i}(t)=d_{i}(t)\left(\sum_{j=1}^{r} d_{j}(t)\right)^{-1}
$$

The geometric validities $\lambda_{i}$ are given by:

$$
\lambda_{i}(t)=\gamma_{i}(t)\left(\sum_{j=1}^{r} \gamma_{j}(t)\right)^{-1}
$$

with:

$$
\gamma_{i}(t)=\left(1-n_{i}(t)\right) \prod_{\substack{j=1 \\ j \neq i}}^{r}\left(1-\exp \left(-\left(\frac{n_{j}(t)}{\sigma}\right)^{2}\right)\right)
$$

$\sigma$ represents a variable regulating parameter with $0<\sigma<0.9$.

The validities $\lambda_{i}$ satisfy the following conditions of convexity:

$0 \leq \lambda_{i} \leq 1$ and $\sum_{i=1}^{r} \lambda_{i}=1$.

The strategy of multiple model control described by geometric approach is given in Figure 1 .

The block "Fusion of elementary controls" aims to calculate the global control $u(t)$ of the fusion of $n$ elementary controls $u_{i}(t)$. 
The global control is driven by the indices of validity $\lambda_{i}(t)$.

In our case, inputs $e$ refers to the state vector $x(t)=\left[x_{1}(t), x_{2}(t), \ldots, x_{n}(t)\right]$ and the desired state vector $x_{d}(t)=\left[x_{1 d}(t), x_{2 d}(t), \ldots, x_{n d}(t)\right]$ of system in the closed loop. These inputs are relevant to the determination of validities.

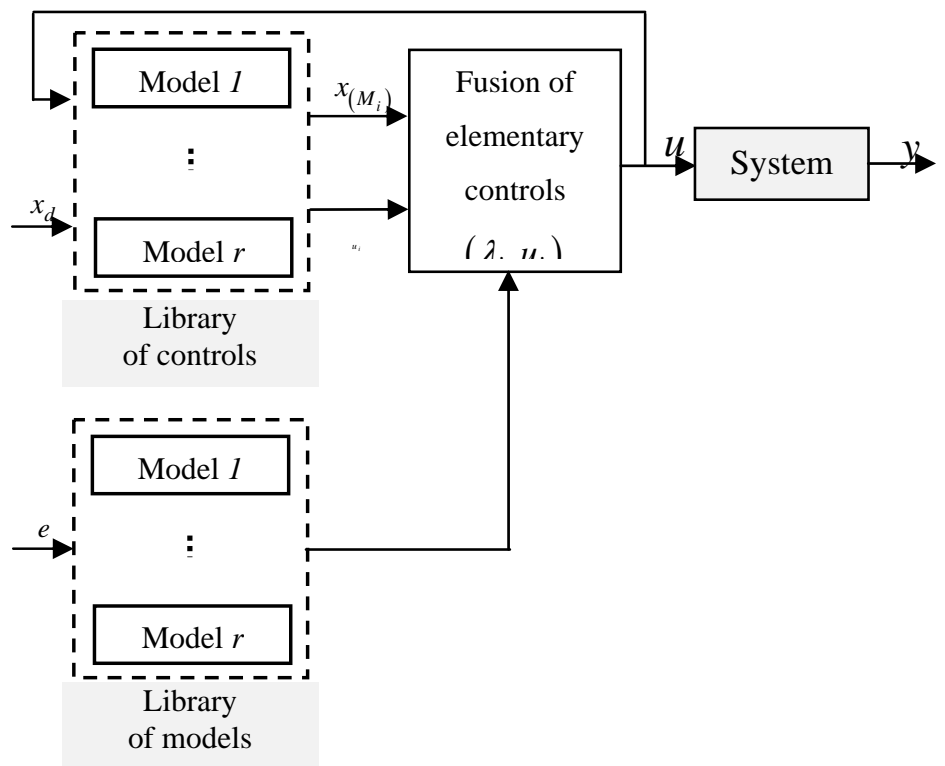

Figure 1. Block diagram of the proposed multiple model control strategy

The global control resulting of the fusion is written as follows:

$$
u(t)=\sum_{i=1}^{r} \lambda_{i}(t) u_{i}(t)
$$

The global output of system is given by:

$$
y(t)=\sum_{i=1}^{r} \lambda_{i}(t) x_{i}(t)
$$

\section{NEW STABILITY CONDITIONS BASED ON STATE FEEDBACK WITH REFERENCE}

The objective in this section is to find from such a formulation LMI matrices state feedback gains $K_{i}$ and those tracking $N_{i}$ for which the state vector $x(t)=\left[\begin{array}{lllll}x_{1}(t) & x_{2}(t) & \ldots & x_{n}(t)\end{array}\right]$ of complex continuous system described by (3) converge to a desired state vector $x_{d}(t)=\left[\begin{array}{llll}x_{1 d}(t) & x_{2 d}(t) & \ldots & x_{n d}(t)\end{array}\right]$, $n$ is the order of the controlled system [5], [9].

For each base model is applied a basic control $u_{i}(t)$ state feedback with reference to the form:

$$
u_{i}(t)=-K_{i} x(t)+N_{i} x_{d}(t)
$$

The global control low $u(t)$ is given by:

$$
u(t)=\sum_{i=1}^{r} \lambda_{i}(t)\left(-K_{i} x(t)+N_{i} x_{d}(t)\right)
$$


The relations (3) and (10) allow to have the representation of multiple model and can be writing as follows:

$$
\left\{\begin{array}{l}
\dot{x}(t)=\sum_{i=1}^{r} \sum_{j=1}^{r} \lambda_{i}(t) \lambda_{j}(t)\left(\left[\begin{array}{ll}
G_{i j} & H_{i j}
\end{array}\right]\left[\begin{array}{c}
x(t) \\
x_{d}(t)
\end{array}\right]\right) \\
y(t)=\sum_{i=1}^{r} \lambda_{i}(t) C_{i} x(t)
\end{array}\right.
$$

with $G_{i j}=A_{i}-B_{i} K_{j}$ and $H_{i j}=B_{i} N_{j}$.

Whether $e(t)$ is the tracking error between the state vector $x(t)$ of continuous system (13) and the desired steady state vector $x_{d}(t)$ with:

$$
e(t)=x(t)-x_{d}(t)
$$

The derivative of the tracking error $\dot{e}(t)=\frac{d e(t)}{d t}$ is written as follows:

$$
\begin{aligned}
\dot{e}(t)= & \sum_{i=1}^{r} \sum_{j=1}^{r} \lambda_{i}(t) \lambda_{j}(t)\left(\left[\begin{array}{ll}
G_{i j} & H_{i j}
\end{array}\right]\left[\begin{array}{c}
x(t) \\
x_{d}(t)
\end{array}\right]\right) \\
& -\dot{x}_{d}(t)
\end{aligned}
$$

For a constant desired steady state vector $x_{d}(t)$, the expression (15) can be written:

$$
\dot{e}(t)=\sum_{i=1}^{r} \sum_{j=1}^{r} \lambda_{i}(t) \lambda_{j}(t)\left(\left[\begin{array}{ll}
G_{i j} & Z_{i j}
\end{array}\right]\left[\begin{array}{c}
e(t) \\
x_{d}(t)
\end{array}\right]\right)
$$

with $Z_{i j}=G_{i j}+H_{i j}$

In case the control matrices $B_{i}$ are square and invertible, there are new stability conditions, formulated in terms of LMI, are developed.

If we used the following constraints:

$$
\left\{\begin{array}{l}
Z_{i j}=0 \quad i, j=1,2, \ldots, r \\
x_{d}(t) \quad \text { constant }
\end{array}\right.
$$

The derivative $\dot{e}(t)$ is written as follows:

$$
\dot{e}(t)=\sum_{i=1}^{r} \sum_{j=1}^{r} \lambda_{i}(t) \lambda_{j}(t) G_{i j} e(t)
$$

Theorem: The equilibrium of the continuous system (10) is globally asymptotically stable if exists a matrix $Q=P^{-1}$ such that $Q=Q^{T}>0$ and square matrices $R_{i}$ with size $n$, such as:

$$
\begin{aligned}
& A_{i} Q-B_{i} R_{i}+(*)<0 \text { for } i=1,2, \ldots, r \\
& \frac{1}{2}\left(A_{i}+A_{j}\right) Q-\frac{1}{2} B_{i} R_{j}-\frac{1}{2} B_{j} R_{i}+(*) \leq 0
\end{aligned}
$$

for $1 \leq i<j \leq r$ 
The gains by stabilizing feedback $K_{i}$ state are calculated by:

$$
K_{i}=R_{i} P \text { for } i=1,2, \ldots, r
$$

The tracking gains $N_{i}$ are given by:

$$
N_{i}=-B_{j}^{-1}\left(A_{j}-B_{j} K_{i}\right) \text { for } i, j=1,2, \ldots, r
$$

Proof: Based on the Lyapunov stability conditions can be written:

$$
\begin{aligned}
& V(e(t))=e^{T}(t) P e(t)>0 \\
& \dot{V}(e(t))=\dot{e}^{T}(t) P e(t)+e^{T}(t) P \dot{e}(t)<0
\end{aligned}
$$

The development of $\dot{V}(e(t))$ gives:

$$
\dot{V}(e(t))=e^{T}(t)\left(\sum_{i=1}^{r} \sum_{j=1}^{r} \lambda_{i}(t) \lambda_{j}(t)\left(P G_{i j}+(*)\right)\right) e(t)<0
$$

From the constraint $Z_{i j}=0$ for $i, j=1,2, \ldots, r$, it follows that:

$$
A_{i}-B_{i} K_{j}+B_{i} N_{j}=0 \text { for } i, j=1,2, \ldots, r
$$

whence the relation (22).

\section{CASE OF STUDY : ELECTRICAL CIRCUIT}

Consider an electric circuit, Figure 2, may models a different dynamic process (thermal, mechanical, hydraulic,...), which includes two resistors $\left(R_{1}, R_{2}\right)$ and two capacitors $\left(C_{1}, C_{2}\right)$ supplied by two voltages $\left(u_{1}(t), u_{2}(t)\right)$.

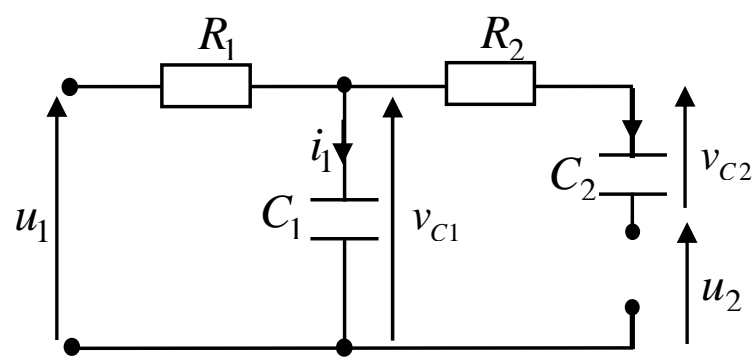

Figure 2. Electrical Circuit

\subsection{System Modeling}

The main objective is to converge $v_{C 1}(t)$ and $v_{C 2}(t)$ to constant values $5 \mathrm{v}$ and $24 \mathrm{v}$ respect.

Following characteristics:

$C_{1}=C_{2}=100 \mu \mathrm{F}, R_{1}=1 \mathrm{~K} \Omega$ and $R_{2}=10 \mathrm{~K} \Omega$.

The differential equations describing the circuit are given by: 


$$
\begin{aligned}
& u_{1}(t)=R_{1}\left(i_{1}(t)+i_{2}(t)\right)+v_{C 1}(t) \\
& v_{C 1}(t)=R_{2} i_{2}(t)+v_{C 2}(t)+u_{2}(t) \\
& \dot{v}_{C 1}(t)=\frac{d v_{C 1}(t)}{d t}=\frac{1}{C_{1}} i_{1}(t) \\
& \dot{v}_{C 2}(t)=\frac{d v_{C 2}(t)}{d t}=\frac{1}{C_{2}} i_{2}(t)
\end{aligned}
$$

By choosing the state vector $x(t)=\left(v_{C 1}(t) \quad v_{C 2}(t)\right)^{T}$ and the control vector $u(t)=\left(u_{1}(t) \quad u_{2}(t)\right)^{T}$, we can write the electric circuit in the following matrix form:

$$
\left\{\begin{array}{l}
\dot{x}(t)=A x(t)+B u(t) \\
y(t)=C x(t)
\end{array}\right.
$$

with:

$$
A=\left(\begin{array}{cc}
-\frac{R_{1}+R_{2}}{R_{1} R_{2} C_{1}} & \frac{1}{R_{2} C_{1}} \\
\frac{1}{R_{2} C_{2}} & -\frac{1}{R_{2} C_{2}}
\end{array}\right), B=\left(\begin{array}{cc}
\frac{1}{R_{1} C_{1}} & \frac{1}{R_{2} C_{1}} \\
0 & -\frac{1}{R_{2} C_{2}}
\end{array}\right) \text { and } C=\left(\begin{array}{ll}
1 & 0 \\
0 & 1
\end{array}\right)
$$

For the construction of model's library, we assume that the resistors $R_{1}$ and $R_{2}$ have an uncertainty such as $\underline{R}_{1} \leq R_{1} \leq \bar{R}_{1}$ and $\underline{R}_{2} \leq R_{2} \leq \bar{R}_{2}$ with: $\underline{R}_{1}=0.8 \mathrm{~K} \Omega, \bar{R}_{1}=1.2 \mathrm{~K} \Omega, \underline{R}_{2}=8.5 \mathrm{~K} \Omega, \bar{R}_{2}=11.5 \mathrm{~K} \Omega$. The four models in the database are defined for combinations $\left(\underline{R}_{1}, \underline{R}_{2}\right),\left(\underline{R}_{1}, \bar{R}_{2}\right),\left(\bar{R}_{1}, \underline{R}_{2}\right)$ and $\left(\bar{R}_{1}, \bar{R}_{2}\right)$. The following matrices $A_{i}$ and $B_{i}$ are calculated:

$$
\begin{aligned}
& A_{1}=\left(\begin{array}{cc}
-13.6765 & 1.1765 \\
1.1765 & -1.1765
\end{array}\right), A_{2}=\left(\begin{array}{cc}
-13.3696 & 0.8696 \\
0.8696 & -0.8696
\end{array}\right), A_{3}=\left(\begin{array}{cc}
9.5098 & 1.1765 \\
1.1765 & -1.1765
\end{array}\right) \text { and } A_{4}=\left(\begin{array}{cc}
-9.2029 & 0.8696 \\
0.8696 & -0.8696
\end{array}\right) . \\
& B_{1}=\left(\begin{array}{cc}
12.5 & 1.1765 \\
0 & -1.1765
\end{array}\right), B_{2}=\left(\begin{array}{cc}
12.5 & 0.8696 \\
0 & -0.8696
\end{array}\right), B_{3}=\left(\begin{array}{cc}
8.3333 & 1.1765 \\
0 & -1.1765
\end{array}\right) \text { and } B_{4}=\left(\begin{array}{cc}
8.3333 & 0.8696 \\
0 & -0.8696
\end{array}\right) .
\end{aligned}
$$

Solving the proposed LMI system stability conditions presented in Theorem, the matrices of feedback gains $K_{i}$ defined in (21) are given by:

$$
K_{1}=\left(\begin{array}{cc}
-0.2666 & -0.6545 \\
-8.3751 & 0.5770
\end{array}\right), K_{2}=\left(\begin{array}{cc}
-0.4467 & -0.4749 \\
-8.3839 & 0.4354
\end{array}\right), K_{3}=\left(\begin{array}{cc}
-0.2534 & -0.6320 \\
-5.8736 & 0.5839
\end{array}\right) \text { and } K_{4}=\left(\begin{array}{cc}
-0.4335 & -0.4525 \\
-5.8769 & 0.4431
\end{array}\right) \text {. }
$$

The matrices of tracking gains $N_{i}$ defined in (22) are given by:

$$
N_{1}=\left(\begin{array}{cc}
0.7334 & -0.6545 \\
-7.3751 & -0.4230
\end{array}\right), N_{2}=\left(\begin{array}{cc}
0.5533 & -0.4749 \\
-7.3839 & -0.5646
\end{array}\right), N_{3}=\left(\begin{array}{cc}
0.7466 & -0.6320 \\
-4.8736 & -0.4161
\end{array}\right), N_{4}=\left(\begin{array}{cc}
0.5665 & -0.4525 \\
-4.8769 & -0.5569
\end{array}\right) \text {. }
$$

\subsection{Simulation Results}

In this section, the proposed control is applied to regulate uncertain non stationary nonlinear systems of electrical circuit described by multiple model approach. In the simulations, we employed Simulink of MATLAB $^{\circledR}$ and the Solver option is “ode45”.

The objective is to stabilize the voltages $v_{C 1}(t)$ and $v_{C 2}(t)$ across the capacitors $C_{1}$ and $C_{2}$ of electrical circuit to constant desired voltages $x_{1 d}=5 \mathrm{v}$ and $x_{2 d}=24 \mathrm{v}$.

The evolutions of state variables $x_{1 i}(t)$ and $x_{2 i}(t)$ for each base model are given in Figures 3 and 4 respectively. 
The performance of trajectory tracking of the state variables $x_{1}(t)$ and $x_{2}(t)$ starting system in closed loop, and desired state variables constant, $x_{1 d}$ and $x_{2 d}$ are illustrated in Figure 5.

Figure 6 shows the evolutions of the voltage $u_{1}(t)$ to the input of electrical circuit and four basic voltages $u_{1 i}(t)$.

Figure 7 shows the evolutions of the voltage $u_{2}(t)$ and four basic voltages $u_{1 i}(t)$.

The state variables $x_{1}(t)$ and $x_{2}(t)$ of the starting system in closed loop converge respectively to desired voltage constants values $x_{1 d}=5 \mathrm{v}$ and $x_{2 d}=24 \mathrm{v}$ with small oscillations (Figure 5). This is a socalled pseudo-periodic oscillations whose amplitude, expressed in volts, is not constant and decreases (The oscillations are damped).
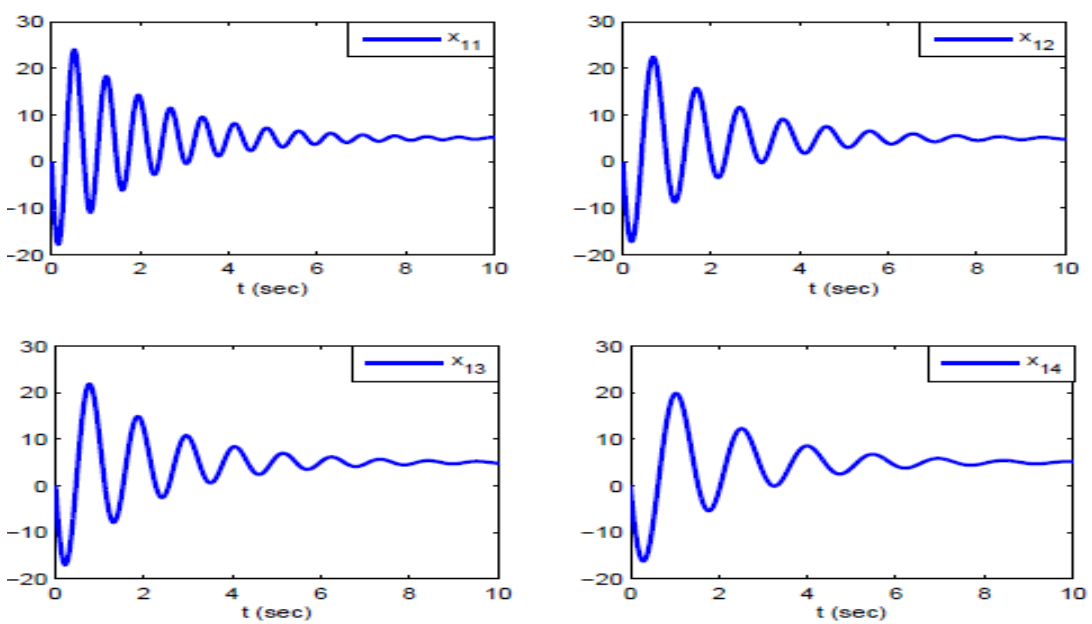

Figure 3. Evolutions of the state variable $x_{1}(t)$ for each model of the base
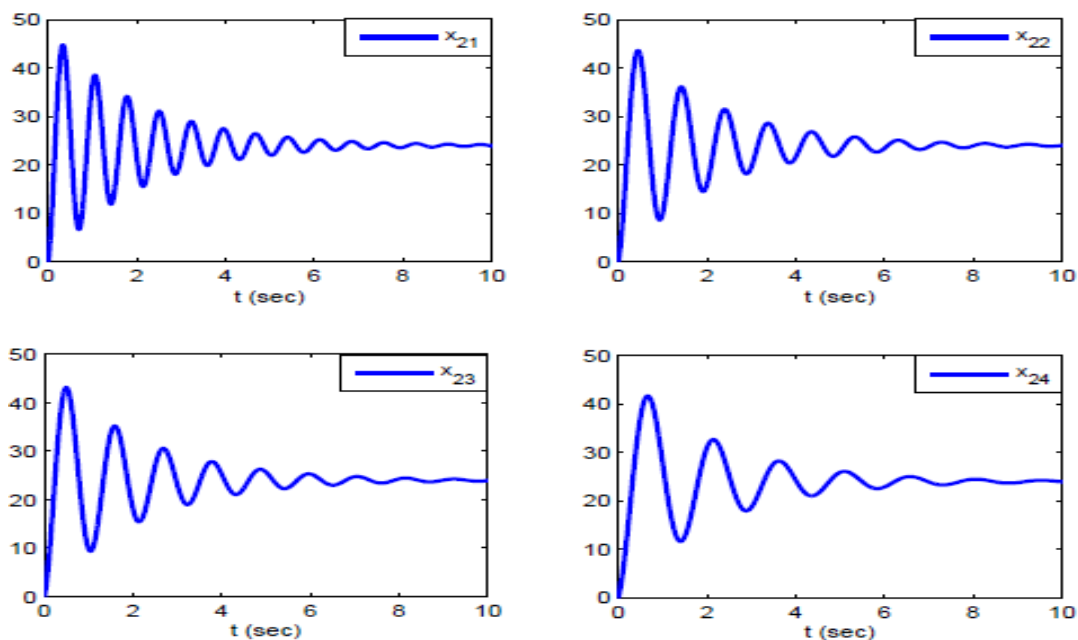

Figure 4. Evolutions of the state variable $x_{2}(t)$ for each model of the base 

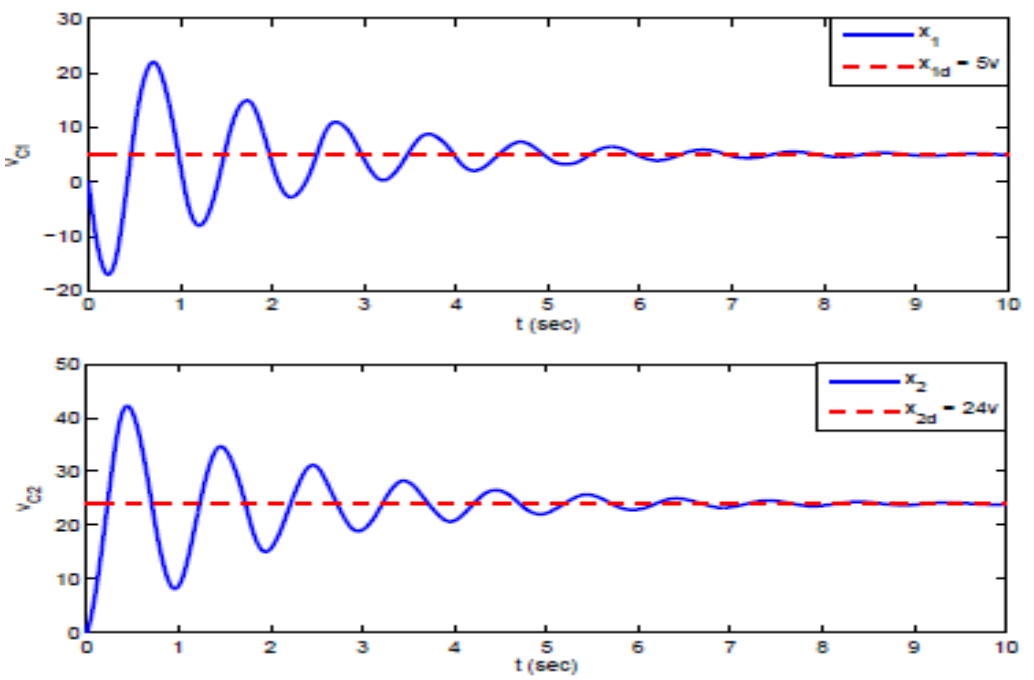

Figure 5. The performance of trajectory tracking of $v_{C 1}(t)$ and $v_{C 2}(t)$ with the desired constant voltages

The multiple model control in which we will be interested consists in the fusion of partial controls. For that we have to compute the validities of each partial model and associate the sub controls weighted by the correspondent coefficients.

The obtained result will control the global process (29).

The global voltages $u_{1}(t)$ and $u_{2}(t)$ are given by the following relations:

$$
\left\{\begin{array}{l}
u_{1}(t)=\sum_{i=1}^{r} \lambda_{i}(t) u_{1 i}(t) \\
u_{2}(t)=\sum_{i=1}^{r} \lambda_{i}(t) u_{2 i}(t)
\end{array}\right.
$$
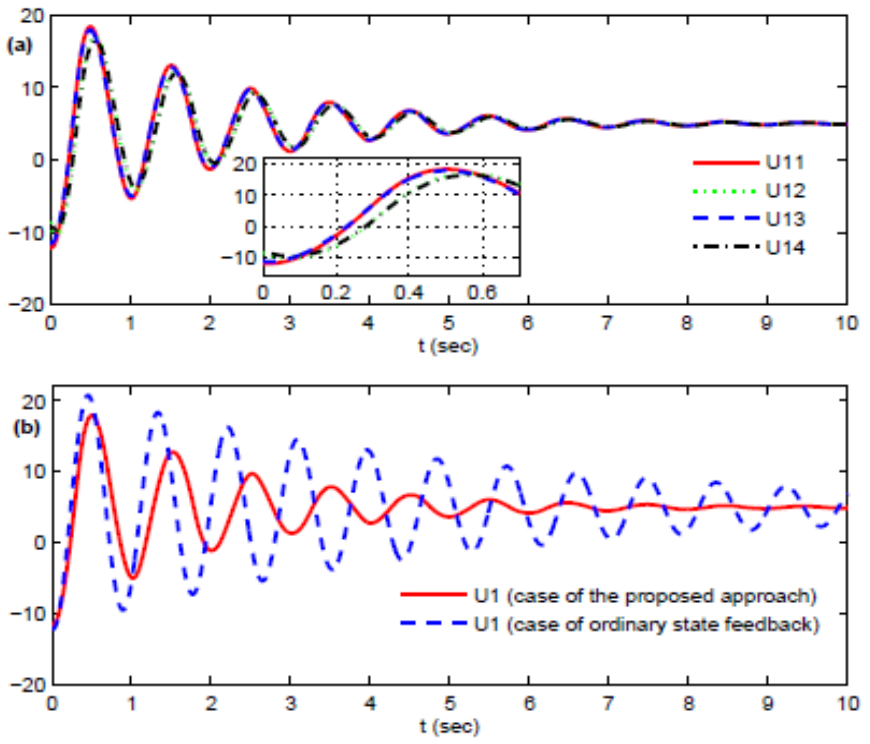

Figure 6. Simulation results of new LMI approach, (a) Evolutions of four basic voltages $u_{1 i}(t)$,

(b) Evolution of the voltage $u_{1}(t)$ with the proposed state feedback with reference 

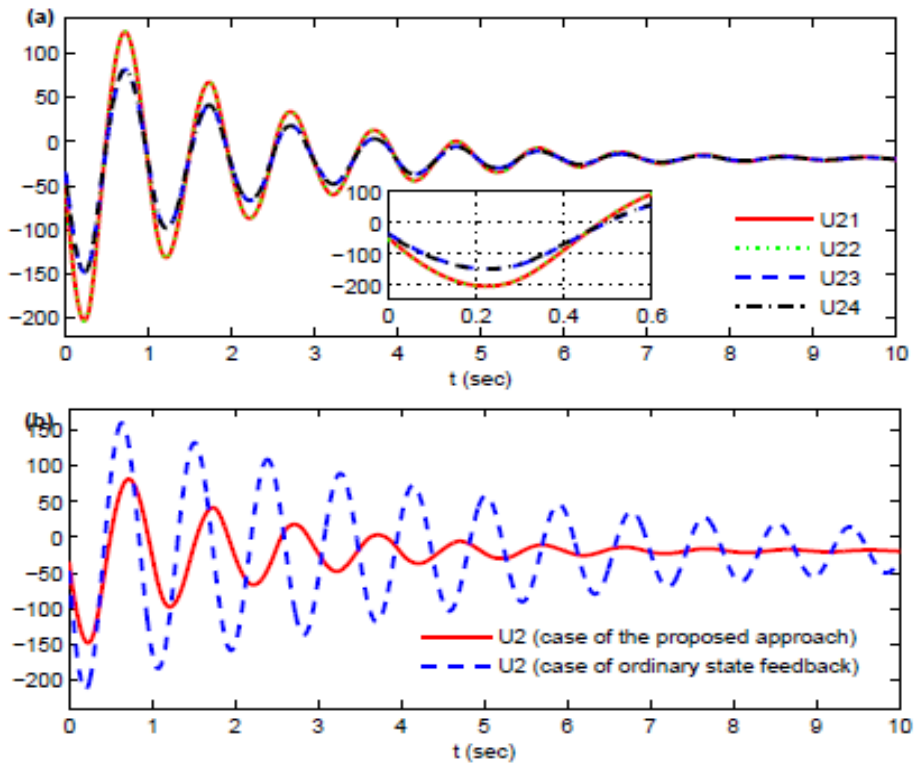

Figure 7. Simulation results of new LMI approach, (a) Evolutions of four basic voltages $u_{2 i}(t)$,

(b) Evolution of the voltage $u_{2}(t)$ with the proposed state feedback with reference

When the new conditions of stability are used, the feedback $K_{i}$ and the tracking $N_{i}$ gains guarantee the stability of the continuous system of electrical circuit, initially unstable, described by the multiple model approach. Thus, all the trajectories of the voltages $v_{C 1}(t)$ and $v_{C 2}(t)$ across the capacitors $C_{1}$ and $C_{2}$ can track the desired constant voltages $x_{1 d}$ and $x_{2 d}$ very well and the global model response is better in the new approach (state feedback with reference) case than in the ordinary state feedback control.

We can conclude that each capacitor discharges and recharges with small oscillations around the value of the desired constant voltages.

\section{CONCLUSION}

In this paper, a contribution in tracking control for a class of continuous non stationary uncertain system with limited parameters is considered. Based on the geometric approach, a library of four models was built. New stability conditions have been developed in the case of multiple model control in the case of state feedback with reference. These conditions allow to reaching the state variables of the closed-loop multiple model describing the complex system starting to the desired state variables. The Matrices gains by state feedback and the matrices of tracking gains are given by solving a system of LMI conditions. Case study of an electrical circuit of second order operative in mode pseudo-periodic is conducted to compare the proposed approach with the ordinary state feedback. Finally, the result of computer simulation has demonstrated the effectiveness of the proposed approach.

\section{REFERENCES}

[1] H. Su, Y. Qu, S. Gao, H. Song and K. Wang, "A model of feedback control system on network and its stability analysis”, Commun Nonlinear Sci Numer Simul, vol. 18, pp. 1822-31, 2013.

[2] M. Ksouri-Lahmari, P. Borne and M. Benrejeb, "Multiple model: the construction of model bases", Studies in Informatics and Control, vol. 3, no. 3, pp. 199-210, 2004.

[3] N. Elfelly, J.Y. Dieulot, M. Benrejeb and P. Borne, "Multimodel control design using unsupervised classifiers", Studies in Informatics and Control, ISSN 1220-1766, vol. 21 (1), pp. 101-108, 2012.

[4] T.A. Johansen, B.A. Foss, E. "Multiple model approaches to modelling and control”, International Journal of Control, Vol. 72, pp. 575, 1999.

[5] H.O. Wang, K. Tanaka and M.F. Griffin, “An approach to fuzzy control of nonlinear systems: stability and design”, issues IEEE Trans. on Fuzzy Syst, vol. 4, no. 4, pp. 14-23, February 1996. 
[6] M.N. Kamarudin, A.R. Husain, M.N. Ahmad and Z. Mohamed, “A Universal Formula for Asymptotic Stabilization with Bounded Controls”, International Journal of Electrical and Computer Engineering (IJECE), ISSN: 20888708, vol. 5, no. 1, pp. 111-118, February 2015.

[7] P. Borne and M. Benrejeb, “On the representation and the stability study of large scale systems”, IJCCC, Baile Felix Spa-Oradea, Romania, pp. 15-17, May 2008.

[8] M. Kannou, "Analyse et synthèse de processus dynamiques continus par approche multimodèle”, $\mathrm{PhD}$ in Automatic Control, National School of En gineers of Tunis, February, 2005.

[9] C. Ghorbel, A. Abdelkrim and M. Benrejeb, "An adaptive fuzzy control of continuous nonlinear systems", 6th International Multi-Conference on Systems, Signals and Devices, SSD, pp. 23-26, Djerba, Tunisia, March 2009.

[10] M. Faieghi, D. Balianu and S. Mashhadi, "Sampled-data nonlinear observer design for chaos synchronization: A Lyapunov-based approach”. Commun Nonlinear Sci Numer Simulat, pp. 2444-2453, vol. 19 (7), 2013.

[11] H. Chapella and S.P. Bhattacharyya, "A generalization of Kharitonov's theorem: Robust stability of interval plants”, IEEE trans. on Automatic Control, vol. 34, no. 3, pp. 306-311, 1989.

[12] M. Chadli, D. Maquin and J. Ragot, “On the stability analysis of multiple model systems”, ECC, Porto, pp.18941899, 2001.

[13] Y.S. Liu, C.H. Fang, "A new LMI-based approach to relaxed quadratic stabilization of T-S fuzzy control systems”, IEEE International Conference on Systems Man and Cybernetics, pp. 2255-2260, 2003.

[14] P. Gahinet, A. Nemirovski, A.J. Laub and M. Chilali, "LMI Control Toolbox, the Math Works”, 1995.

[15] C.W. Chen, "Stability conditions of fuzzy systems and its application to structural and mechanical systems", Advances in Engineering Software, vol 37, pp. 624-629, 2006.

[16] M. Johansson, A. Rantzer and K. Arzen, “Piecewise quadratic stability for affine Sugeno systems”, Fuzzy IEEE’98, Anchorage, Alaska, 1998.

[17] A. Kruszewski, R.Wang and T.M. Guerra, "Nonquadratic stabilization conditions for a class of uncertain nonlinear discrete time TS fuzzy models: a new approach”, IEEE Transactions on Automatic Control, vol. 53, no. 2 , pp. 606611, 2008.

[18] F. Delmotte, L. Dubois, P. Borne, “A General Scheme for Multi-Model Controller using Trust”, Mathematics and Computers in Simulation, vol. 41, pp. 173-186, 1996.

[19] A. Sassi, C. Ghorbel and A. Abdelkrim, "Multiple model adaptive control of complex systems", International Conference on Control, Engineering and Information Technology, pp.229-235, 2014.

[20] F. Pirouzmand, "Robust Model Predictive Control Based on MRAS for Satellite Attitude Control System", International Journal of Electrical and Computer Engineering (IJECE), ISSN: 2088-8708, vol. 4, no. 1, pp. 81-92, February 2014.

[21] S. Boyd, L. El Ghaoui, E. Feron and V. Balakrishann, "Linear matrix inequalities in system and control theory", SIAM, Philadelphia, PA, 1994.

[22] C.H. Fang, Y.S. Liu, S.W. Kau, L. Hong, C.H. Lee, "A new LMI-based approach to relaxed quadratic stabilization of T-S fuzzy control systems”, IEEE Transactions on Fuzzy Systems, vol. 14, no. 3, pp. 386-397, 2006.

[23] A. Abdelkrim, C. Ghorbel and M. Benrejeb, "LMI-based tracking control for Takagi-Sugeno fuzzy model”, International Journal of Control and Automation, vol. 3, no. 2, pp. 21-36, June 2010.

\section{BIOGRAPHIES OF AUTHORS}

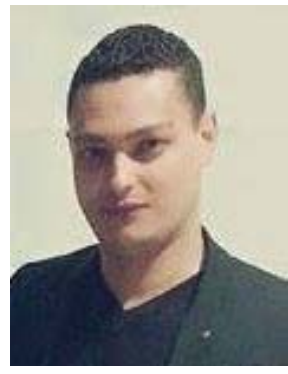

Ameur Sassi received his Master degree in Automatic Control and Signal Processing in 2012 from the National School of Engineers of Tunis (ENIT). He is currently preparing the $\mathrm{PhD}$. degree in Electrical Engineering within the framework of research laboratory in Automatic Control LA.R.A ENIT. His research is related to analysis and synthesis of complex systems described by multiple model approach and synthesis of control laws of dynamic systems.

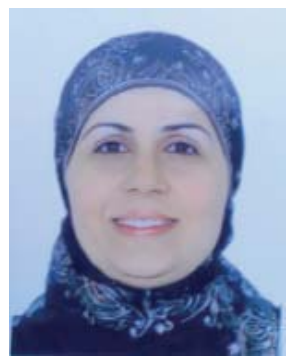

Afef Abdelkrim has obtained a Diploma of Engineer in 2000 and the $\mathrm{PhD}$. in Electrical Engineering at the National School of Engineers of Tunis (ENIT) in 2005. She is currently Senior Lecturer at the Departement of Electrical Engineering of the High School of Technology and Computer Science (ESTI). Her research interests are in handwriting process modelling and synthesis and in the artificial neuronal network domains. 